\title{
Quasi-particles dynamics in underdoped Bi2212 under strong optical perturbation
}

\author{
Giacomo Coslovich $^{*}$, Claudio Giannetti ${ }^{\dagger}$, Federico Cilento ${ }^{\dagger}$, Gabriele \\ Ferrini $^{\dagger}$ and Fulvio Parmigiani*** \\ ${ }^{*}$ Department of Physics, Università degli Studi di Trieste, Trieste I-34127, Italy \\ ${ }^{\dagger}$ Department of Physics, Università Cattolica del Sacro Cuore, Brescia I-25121, Italy \\ ${ }^{* *}$ Elettra Synchrotron of Trieste, Basovizza I-34127, Italy
}

\begin{abstract}
.
In this work an optical pump-probe set-up is used to study the photo-induced non-equilibrium dynamics of a superconducting underdoped Bi2212 single crystal in a strong excitation regime $\left(10<\phi<600 \mu \mathrm{J} / \mathrm{cm}^{2}\right)$. The use of a tunable repetition rate $120 \mathrm{fs}$ pulsed laser source allows us to avoid significant average heating of the sample and to optimize the signal-to-noise ratio in the detection of the transient reflectivity variation. A discontinuity of the transient reflectivity is observed at high excitation intensities $\left(\phi \sim 70 \mu \mathrm{J} / \mathrm{cm}^{2}\right)$. Numerical simulations of the heat diffusion problem indicate that, in this regime, the local temperature of the sample is lower than $T_{C}$, confirming the impulsive nature of this phenomenon. The quasi-particles $(\mathrm{QP})$ dynamics in the strongly perturbed superconducting state $\left(10<\phi<70 \mu \mathrm{J} / \mathrm{cm}^{2}\right)$ is analysed within the framework of the RotwarfTaylor model. The picture emerging from the data is consistent with a dynamics governed by highfrequency phonon (HFP) population, which causes a "bottleneck" effect in the QP recombination.
\end{abstract}

Keywords: HTSC, Copper compounds, Non-equilibrium superconductivity, Photoexcitation PACS: 74.25.Gz, 74.40.+k, 74.72.Hs, 78.47.+p

\section{INTRODUCTION}

Since their discovery in 1986 the efforts to understand the pairing mechanism in highcritical temperature superconductors (HTSC) have been intense. From the experimental point of view, the HTSC have been widely studied with tecniques such as angle-resolved photoemission spectroscopy (ARPES) [1], scanning tunneling spectroscopy (STS) [2] and optical spectroscopy [3]. These experiments gave new insights into the superconducting gap symmetry and the universal phase diagram of HTSC. However the main question concerning the origin of the pairing mechanism remains open. $[4,5,6]$

In the last decades, the non-equilibrium dynamics in HTSC have been widely investigated by means of femtosecond pump-probe spectroscopy $[7,8,9,10,11,12,13,14$, $15,16,17,18,19,20,21]$. In these experiments the system is brought out of equilibrium by an ultra-short laser pulse ("pump") and the transient variation of the optical properties is studied at a variable delay time by means of a second laser pulse ("probe"). In the weak perturbation regime the number of excitations photoinjected by the laser pulse is negligible as compared to the thermal excitations and the equilibrium energy spectrum of the system can be assumed. In this regime, the decay dynamics of the excitations can be described by simple rate equations containing information on the pairing mechanism. The main issue addressed in these experiments was to elucidate whether 
the cuprates are in the so called "phonon bottleneck" regime, i.e. the dynamical regime that occurs in conventional superconductor, where the relaxation of QP is slowed down by the high-frequency phonon (HFP) relaxation rate. As proposed earlier by Rothwarf and Taylor [22] such dynamics is the manifestation of a quasi-equilibrium between QP and HFP, and can be represented by a set of two coupled differential equations, the Rothwarf-Taylor equations, (RTE). The search of a "phonon bottleneck" in the cuprates was guided by the idea that this finding would clarify the relationship between Cooper Pair (CP) formation and a phonon-mediated interaction between electrons. Nevertheless pump-probe experiments have not yet clarified this point, since the observed dynamics is compatible both with the RTE in the strong bottlenck regime [17] and with a bimolecular recombination process $[20,11]$. The latter interpetation being a possible signature of a purely electronic process behind the $\mathrm{CP}$ formation.

The strong perturbation regime, i.e. when the density of the photoinjected excitation strongly perturb the superconducting condensate and the energy spectrum of the system, has been scarcely investigated for problems related to the average heating of the sample. The bulk of experimental work in this field has been perfomed in the low intensity regime, with pump fluence $\phi<10 \mu \mathrm{J} / \mathrm{cm}^{2}$. This is related to the wide use of high repetition rate Ti:Sa oscillator $(\approx 80 \mathrm{MHz})$ which may cause serious steady-state heating effects on highly anisotropic HTSC single crystals [12]. On the other hand, low repetition rate amplified sources (typically $1 \mathrm{kHz}$ ) are suitable to work at very high intensities, but with the drawback of a lower statistics. At very high excitation fluences $\left(\phi>1 \mathrm{~mJ} / \mathrm{cm}^{2}\right)$ the superconducting state exhibits the same relaxation of the normal state $[10,23]$. This behaviour can be attributed to a photo-induced phase transition (PIPT) to the normal state which has been observed in the past in conventional superconductors [24, 25, 26].

However a time-resolved study, with sub-ps time resolution, of the crossover from the low to the high-intensity regime is still lacking. In particular the investigation of the QP dynamics at intensities just below this crossover is of core importance in understanding non-equilibrium superconductivity in HTSC. To address this question the two critical experimental parameters are the effective temperature of the sample area in the laser spot and the signal-to-noise ratio of the detection. The use of a tunable repetition rate laser source is mandatory to obtain the complete control of these parameters.

In this work we perform a femtosecond optical pump-probe study of the relaxation dynamics of $\mathrm{Bi}_{2} \mathrm{Sr}_{2} \mathrm{CaCu}_{2} \mathrm{O}_{8+\delta}$ under strong excitation $\left(10<\phi<600 \mu \mathrm{J} / \mathrm{cm}^{2}\right)$ using a Cavity Dumped Ti:Sapphire oscillator (repetition rate ranging from $54 \mathrm{MHz}$ to single shot). A detailed study of the heating effects, supported by both experimental observation and numerical simulation, is here reported. We show that we can optimize the signal-to-noise ratio, while minimizing the local average heating.

The QP dynamics at fluences just below the crossover for the PIPT is discussed in the framework of RTE. From this analysis we conclude that the energy is mainly absorbed by HFP which consitutes a "strong bottlneck" for the QP recombination.

\section{EXPERIMENTAL METHODS}

Pump-probe measurements have been performed on an under-doped $\mathrm{Bi}_{2} \mathrm{Sr}_{2} \mathrm{CaCu}_{2} \mathrm{O}_{8+\delta}$ (Bi2212) single crystal. At this doping level the compound exhibits three phases, the 


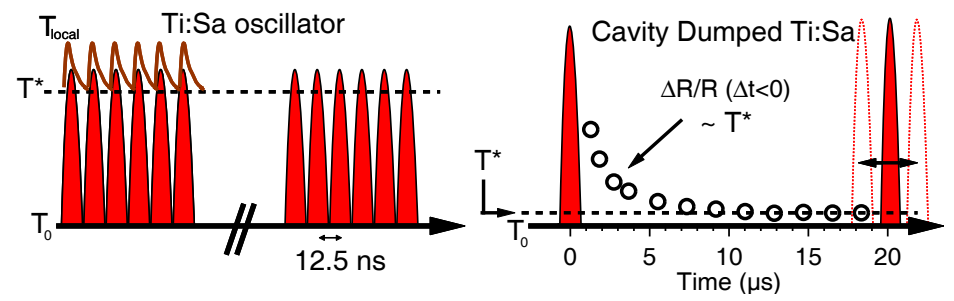

FIGURE 1. Cavity Dumped Ti:Sapphire set-up scheme: on the left panel a sketch of the local temperature on the laser spot, $\mathrm{T}_{\text {local }}$, is shown in the case of a Ti:Sa oscillator (repetition rate $80 \mathrm{MHz}$ ) on the timescale of ns. $\mathrm{T}_{0}$ is the cold finger temperature, while $\mathrm{T}^{*}$ is the effective temperature in the laser spot at $\mathrm{t} \lesssim 0 . \Delta \mathrm{T}^{*}=\mathrm{T}^{*}-\mathrm{T}_{0}$ is the average heating. On the right the sketch refers to the Cavity Dumped Ti:Sa oscillator, thus the time spacing between pulses is tunable (see dotted pulse shape). We report (black circles) the value of $\Delta \mathrm{R}(\mathrm{t} \leqslant 0) / \mathrm{R}$, i.e., just before the arrival of each pulse, which is an indicator of $\Delta \mathrm{T}^{*}$. When the repetition rate is lowered the measure corresponds to a longer time delay between pulses and $\Delta \mathrm{T}^{*}$ decreases. The measurement is taken at $\mathrm{t}=-1 \mathrm{ps}, \mathrm{T}_{0}=130 \mathrm{~K}$ and $\phi \sim 600 \mu \mathrm{J} / \mathrm{cm}^{2}$.

normal state ( $\left.\mathrm{T}>\mathrm{T}_{P G} \sim 160 \mathrm{~K}[1]\right)$, the pseudo-gap state, where an energy-gap appears in the low-energy excitation spectrum of the system $\left(\mathrm{T}_{C}<\mathrm{T}<\mathrm{T}_{P G}\right)$, and the superconducting one $\left(\mathrm{T}<\mathrm{T}_{C}=81 \mathrm{~K}\right)$. The value of $\mathrm{T}_{C}$ was tested with $\mathrm{AC}$ magnetic susceptibility measurements, giving an amplitude of transition of $\pm 2 \mathrm{~K}$. Moreover sample composition, homogeneity and c-axis orientation dispersion were checked by means of X-Ray diffraction on both bulk sample and its powder. Results confirmed the absence of other structural phases of the compound and a lattice costant along the c-axis of $3.09 \mathrm{~nm}$ with an angular dispersion of $1 \mathrm{deg}$.

In our experiments the pump and probe beams were produced using a cavity dumped Ti:Sapphire oscillator. The output of this system is a train of $120 \mathrm{fs}$ lasers pulses, with $1.5 \mathrm{eV}$ photon energy and an adjustable repetition rate ranging from $54 \mathrm{MHz}$ to single shot (see FIG.1). The pulse energy output is $\sim 55 \mathrm{~nJ}$ at $108 \mathrm{kHz}$ repetition rate. The radiation is split into the pump and the probe beams by means of a $30 \%-70 \%$ beam splitter. The pump pulse is then delayed through a fast-moving delay line and modulated in intensity at $3 \mathrm{kHz}$ by a mechanical chopper. Both the beams are then focused on the sample surface. The diameters of pump and probe spots on the surface are respectively 45 and $30 \mu \mathrm{m}$, the latter being sufficiently smaller to probe the optical properties of a uniformly excited area. The variation of the reflectivity is acquired through a lock-in amplifier, referenced to the mechanical chopper. A signal-to-noise ratio of the order of $10^{-5}$ is obtained at a repetition rate of $108 \mathrm{kHz}$.

\section{RESULTS}

The transient reflectivities $\Delta R(t) / R$ in the three phases of underdoped Bi2212 at a repetition rate of $54 \mathrm{kHz}$ are summarized in FIG.2. At low fluence (red lines, $\phi \sim 50 \mu \mathrm{J} / \mathrm{cm}^{2}$ ) the three dynamics are different, in agreement with previous results in the literature [21]. The normal state response can be interpreted within an extended three-temperature model [27] and is characterized by an initial electron-phonon relaxation time of $470 \mathrm{fs}$ (see FIG.2). The transient reflectivity abruptly changes sign below the temperature as- 


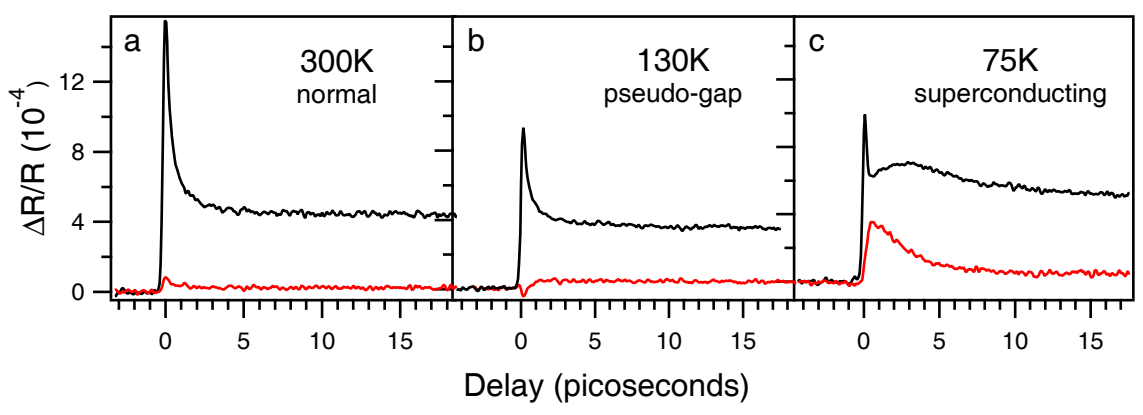

FIGURE 2. Transient optical response at high $\left(\phi \sim 600 \mu \mathrm{J} / \mathrm{cm}^{2}\right.$, black lines) and low fluences $\phi \sim 50$ $\mu \mathrm{J} / \mathrm{cm}^{2}$, red lines) in the normal (a), pseudogap (b) and superconducting (c) phases of Bi2212. The initial decays of (a) and (b) can be fitted with an exponential function convolved with the laser pulse shape. The fits for the high fluence data give for the initial relaxation times, $\tau=470 \mathrm{fs}$ in (a) and $\tau=440 \mathrm{fs}$ in (b).

sociated with the appearence of the so-called pseudo-gap. Here the relaxation is slightly faster, with a characteristic time of $\sim 300$ fs. When the temperature is decreased below $\mathrm{T}_{C}, \Delta R / R$ becomes positive with a maximum variation one order of magnitude larger than the normal state one. The relaxation time increseas up to $\tau \sim 2-3 \mathrm{ps}$.

Black lines in FIG. 2 are the transient reflectivity at high fluence $\left(\phi \sim 600 \mu \mathrm{J} / \mathrm{cm}^{2}\right)$. We notice that the pseudo-gap response is now similar to that of the normal state, and the signal in the superconducting state has a novel two component dynamics. In the following, we argue that the measured two-component dynamics at high-fluences $(\phi>70$ $\mu \mathrm{J} / \mathrm{cm}^{2}$ ) is a genuine impulsive effect, not related to the average local heating of the sample.

In pump-probe experiments there are three different timescales: the first is related to the impulsive heating of the electronic system on the same scale of a single laser pulse duration (120 fs); the second is caused by the impulsive heating of the lattice, occurring on the timescale of the electron-phonon coupling ( $400 \mathrm{fs}$ ) [28, 29]; the third is the relaxation on the nanosecond timescale because of the heat conduction through the sample (see FIG.1). If the time spacing between subsequent pulses is too short in respect to this last timescale, the repeated pulses may cause the average heating of the pumped area. The first two sub-ps dynamics are proportional to the energy per pulse and are a genuine non-equilibrium effect, whereas the last is related to the mean intensity of the laser and is a spurious effect in pump-probe experiments.

The possibility to tune the laser repetition rate, keeping constant the energy per pulse allowed us to disentangle the non-equilibrium dynamics from the steady-state heating and to minimize this effect. FIG.1 sketches the behaviour of the local average temperature $\mathrm{T}^{*}$ at different repetition rates. $\mathrm{T}^{*}$ can be estimated by measuring the reflectivity variation at negative delays, i.e. before the arrival of the pump pulse. Under the experimental condition reported in FIG.1 we observe that a repetition rate as low as $200 \mathrm{kHz}$ (corresponding to a time delay of $5 \mu$ s between pulses) must be used to significantly decrease the average heating.

In order to quantitatively evaluate $\mathrm{T}^{*}$, we performed time-resolved reflectivity measurements at different repetition rates in the superconducting state at a temperature very 


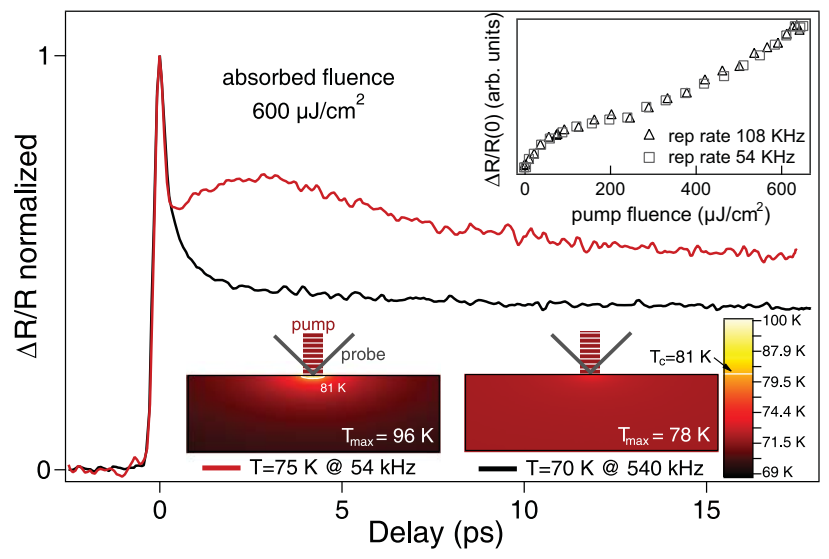

FIGURE 3. Time-resolved reflectivity measurements at $54 \mathrm{kHz}$ and $540 \mathrm{kHz}$ repetition rates are reported. The calculated spatial profiles of the temperature increase for both repetition rates are reported in the image plot. At $540 \mathrm{kHz}$ repetition rate and $\mathrm{I}_{\text {pump }}=600 \mu \mathrm{J} / \mathrm{cm}^{2}$, the temperature of the probed volume exceeds $\mathrm{T}_{C}=81 \mathrm{~K}$ (white line), whereas at $54 \mathrm{kHz}$ the average temperature is limited to $78 \mathrm{~K}$. The thermal conductivities are the T-dependent data obtained from $[31,32]$. The values at $\mathrm{T}=70 \mathrm{~K}$ are $\kappa_{c}=0.65 \mathrm{~W} / \mathrm{mK}$ and $\kappa_{a b} 4.6 \mathrm{~W} / \mathrm{mK}$. (c) $\Delta \mathrm{R} / \mathrm{R}(\mathrm{t}=0)$ as a function of the pump fluence, at different repetition rates.

close to $\mathrm{T}_{C}(\mathrm{~T} \sim 75 \mathrm{~K})$. In this regime, a small change in $\mathrm{T}^{*}$ can be easily measured since the optical response of a superconductor abruptly changes at $\mathrm{T}_{C}[14,21,30,9]$. In particular we focused on the high-fluence regime $\left(\phi \sim 600 \mu \mathrm{J} / \mathrm{cm}^{2}\right)$, where the average heating effects are more serious. In FIG. 3 we show that, at a repetition rate of $54 \mathrm{kHz}$, the normalized signal exhibits the coexistence of a fast and a slow decay. At a repetition rate of $540 \mathrm{kHz}$, we observe a single fast decay, similar to the decay observed in the normal state $(\mathrm{T}=300 \mathrm{~K})$. As a consequence, we argue that at $54 \mathrm{kHz}$ the local temperature $\mathrm{T}^{*}$ is below $\mathrm{T}_{C}$, whereas at $540 \mathrm{kHz} \mathrm{T}^{*}$ overwhelms $\mathrm{T}_{C}$ and the superconductivity is thermally quenched.

To further exclude any role of the average heating, we reported in the inset of FIG. 3 the behavior of $\Delta R / R$ at zero delay, as a function of the intensity. The measured nonlinearity is the evidence of the discontinuity of the optical response at high fluences [30]. The result that the non-linear behavior is independent of the repetition rate confirms that the high-intensity regime is not related to the average intensity impinging on the sample. Above $200 \mathrm{kHz}$ the average heating can not be prevented.

In order to confirm our results, we perfomed numerical simulations of the 3D heat diffusion problem for the case of a $5 \times 5 \times 0.5 \mathrm{~mm}^{3} \mathrm{Bi} 2212$ sample using a finite element analisys software (COMSOL). In the simulations, we used the T-dependent values of the thermal conductivity and specific heat reported in the literature [31, 32, 34]. Both pump and probe beams were considered as heat sources. The asymptotic temperature distribution $\mathrm{T}^{*}(\mathrm{x}, \mathrm{y}, \mathrm{z})$ was obtained from simulations (see image plots in FIG.3) and the value in the center of both pump and probe beams, $T^{*}(0,0,0)$, is the estimated upper limit of $\mathrm{T}^{*}$. Numerical simulations give consistency to our experimental findings. In fact at a repetition rate of $540 \mathrm{kHz}$ the temperature of the whole probed area increases from 70 $\mathrm{K}$ to $\mathrm{T}^{*}>\mathrm{T}_{C}=81 \mathrm{~K}$, with a maximum of $96 \mathrm{~K}$, whereas at $54 \mathrm{kHz}$ the maximum $\Delta \mathrm{T}^{*}$ is 3 $\mathrm{K}$. 
Since thermal conduction in the Bi2212 is strongly temperature dependent and highly anisotropic [31], average heating effects depend on the initial sample temperature $\mathrm{T}_{0}$. In particular when the temperature is lowered well below $\mathrm{T}_{C}$ both c-axis and in-plane thermal conductivities abruptly decrease [31, 32], causing an higher $\Delta \mathrm{T}^{*}$. Thus the common method $[15,11,33]$ of checking the variation of the $\mathrm{T}_{C}$ under the laser spot, e.g., by monitoring the local resistance [15], is not sufficient to correctly estimate the laser heating at $\mathrm{T} \ll \mathrm{T}_{c}$ in the high fluence regime. We stress that, from our simulations, $\mathrm{T}^{*}(\mathrm{x}, \mathrm{y}, \mathrm{z})$ can be calculated at any temperature and at any laser intensity. This allow us to obtain the correct local temperature in the laser spot and thus to select the proper repetition rate which gives the best compromise between signal-to-noise and average heating for a particular fluence, temperature and sample.

In conclusion, we demonstrated that the drastic change in the optical response of superconducting Bi2212 (FIG. 2 c), at high-fluences, is not related to the average heating of the excited area, but it is a genuine non-equilibrium effect.

\section{DISCUSSION}

The transient reflectivity dynamics of underdoped Bi2212 in our experimental excitation range $\left(10<\phi<600 \mu \mathrm{J} / \mathrm{cm}^{2}\right)$ exhibits a discontinuity above an intensity threshold $\left(\phi_{t h} \sim 70\right.$ $\mu \mathrm{J} / \mathrm{cm}^{2}$ ), related to the PIPT to the normal state [30]. Here we focus on the QP dynamics in the superconducting state just below $\phi_{t h}$. In FIG. 4 we report $\Delta \mathrm{R} / \mathrm{R}(\mathrm{t})$ at $\phi=40 \mu \mathrm{J} / \mathrm{cm}^{2}$. We choose the optimal repetition rate $(540 \mathrm{kHz})$ in order to have a sufficient signal-tonoise ratio and a $\Delta \mathrm{T}^{*}<3 \mathrm{~K}$. The temperature of the cold finger is $\mathrm{T}_{0}=22 \mathrm{~K}$, while the estimated local temperature is thus $\mathrm{T}^{*} \sim 25 \mathrm{~K}$. This approach allows us to explore a new regime of the QP recombination in HTSC, namely the strong perturbation regime, where the superconducting condensate is perturbed by a number of photo-excited carriers much higher than the thermal excited ones but it is not yet destroyed by them.

The recombination dynamics in both conventional and HTSC has been successfully interpreted using the RTE [22, 17]

$$
\left\{\begin{array}{l}
\dot{n}=I_{q p}(t)+2 \gamma p-\beta n^{2} \\
\dot{p}=I_{p h}(t)+\beta n^{2} / 2-\gamma p-\gamma_{e s c}\left(p-p_{T}\right)
\end{array}\right.
$$

describing the surface density of QP, $n$, and HFP, $p . n$ and $p$ are in general a sum of thermal excitations (respectively $n_{T}$ and $\left.p_{T}\right)$ and photo-induced excitations $\left(n_{P I}\right.$ and $\left.p_{P I}\right)$. The coupling between $n$ and $p$ is obtained through a) the annihilation of a $\mathrm{CP}$ via HFP absorption and the creation of two QP ( $\gamma p$ term) and $b)$ the emission of HFP during the two-body direct recombination of QP to form a CP $\left(\beta n^{2}\right.$ term). In this model, the total population decreases through the $\gamma_{\text {esc }}$ term in Eq.(1), which is determined by two processes: the escape of non-equilibrium HFP from the probed region and their energy relaxation through inelastic scattering with thermal phonons. When $\gamma \gg \gamma_{e s c}$ the decay dynamics is in the so-called phonon bottleneck regime, and the relaxation is ultimately regulated by the escape rate of the non-equilibrium $\operatorname{HFP}\left(\gamma_{e s c}\left(p-p_{T}\right)\right.$ term). This regime has been observed in conventional superconductors $[22,16,19]$, where the measured

QP decay time is of the order of nanoseconds, the typical time for anharmonic decay of acoustic phonons of some meV. 


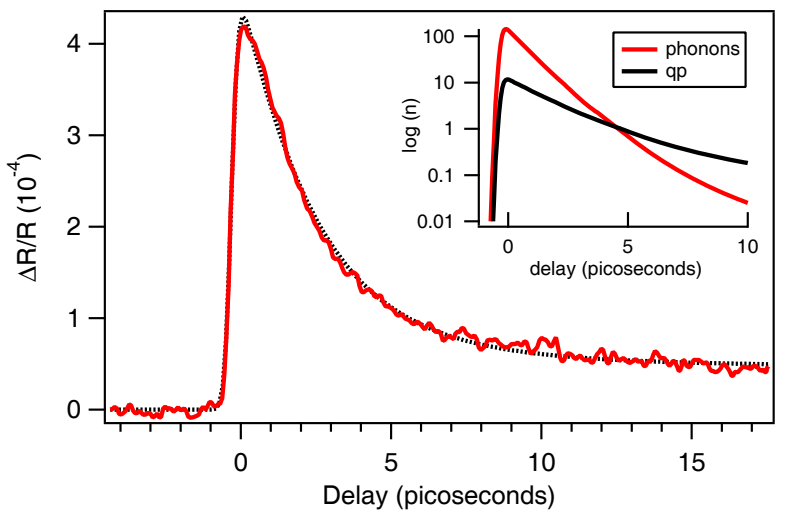

FIGURE 4. Time-resolved reflectivity measurement in the superconducting state $\left(\mathrm{T}^{*}=25 \mathrm{~K}\right)$, with pump fluence $\phi=38 \mu \mathrm{J} / \mathrm{cm}^{2}$. The black dotted line is the fit with numerical solution of the RTE (fitting parameter in the text). In the inset the number densities of HFP and QP are reported in a log scale.

In HTSC the situation is subtler, since the lifetime of HFP is close to the intrinsic QP recombination time. Moreover a theoretical estimation of $\gamma$ and $\gamma_{e s c}$ gives the same order of magnitude [18]. Thus it is not easy to discriminate between a dynamics governed by the RTE in the phonon bottleneck regime or by a bi-molecular recombination process. In particular, a strong temperature and pump intensity dependence has been observed for underdoped Bi2212 and YBCO, which could be explained both by the RTE in the low-T regime [17] and by a bi-molecular dynamics with $\beta \approx 0.1 \mathrm{~cm}^{2} / \mathrm{s}$ [11].

In our measurements, the strong perturbation regime $\left(n_{P I}>n_{T}\right)$ of the RTE is attained. At $\phi \sim 40 \mu \mathrm{J} / \mathrm{cm}^{2}$, the photo-induced QP density, $n_{P I}$, is $\sim 10^{21} \mathrm{~cm}^{-3}$, which is several order of magnitude higher than $n_{T}$ for $\mathrm{T}$ far enough from $\mathrm{T}_{C}$. As a consequence, the thermal contribution to the excitations numbers, $n_{T}$ and $p_{T}$, becomes negligible. In this case, the equations cannot be linearized as in the case of low-excitation $[11,17]$ and a numerical solution of the non-linear system is needed. We thus fitted our transient reflectivity signal with the numerical solution of the RTE, Eq. 1 , considering $n \equiv n_{P I}$ and $p \equiv p_{P I}$. In the fitting procedure we fixed the parameter $\beta$ as reported in the literature $\beta=0.1 \mathrm{~cm}^{2} / \mathrm{s}$ [11]. To set the proper initial condition in the RTE one should consider the fact that the laser pulse creates high-energy QP of $1.5 \mathrm{eV}$ which subsequently relaxes to gap-energy excitations ( $n$ and $p$ ). In the normal state the QP multiplication process (electron thermalization) is fast ( $\tau_{e-e} \sim 10 \mathrm{fs}$ ) and the maximum is reached within the pulse duration (see FIG. 2 a). On the contrary in the superconducting state the rise time is slower, meaning that quasi-equilibrium between $n$ and $p$ is reached on a timescale longer than the pulse duration. The fact that the rise time is very close to the normal state e-ph relaxation time, $\tau_{e-p h}$, suggests that the initial population is dominated by $\mathrm{HFP}$, in agreement with the case of coventional superconductors, e.g. $\mathrm{MgB}_{2}$ [16]. Thus we fixed $I_{p h}(t)$ such that all the pump energy $(1.5 \mathrm{eV} \times$ number of photons $)$ is absorbed by $40 \mathrm{meV}$ HFP. The time duration of $I_{p h}(t)$ was assumed equal to the e-ph relaxation time in the normal state, $\tau_{e-p h}=470 \mathrm{fs}$.

The numerical solution of the RTE reproduces the experimental QP dynamics (see FIG. 4). We are able to determine the parameter $\gamma_{e s c}=0.8 \pm 0.1 \mathrm{ps}^{-1}$, while for the 
parameter $\gamma$ we obtain a lower bound, i.e. $\gamma \gtrsim \gamma_{e s c}$. In fact when $\gamma \gtrsim \gamma_{e s c}$ the QP rise time is the same of $I_{p h}(t)$, i.e. 470 fs (see fit in FIG. 4), whereas when $\gamma_{\ll} \gamma_{e s c}$ the rise time increases and the dynamics is no more compatible with the measured one. The obtained values are compatible with recent experiments on LSCO [35] and theoretical estimation of anharmonic processes in YBCO [18]. These findings suggest that the laser energy is mainly absorbed by HFP, as we can see in the inset of FIG. 4. This is in agreement with the theoretical predictions of the $\mathrm{T}_{\text {eff }}$ model for non-equilibrium superconductivity [36] and $\mathrm{THz}$ experiments on $\mathrm{MgB}_{2}$ [16]. Thus the HFP play an important role in the excitation regime where a discontinuity of the optical response is observed [30]

\section{CONCLUSION}

In this paper, the ultrafast optical response dynamics of underdoped Bi2212, in a previously unexplored excitation intensity regime, is measured and discussed. We first demostrated that our experimental set-up allows the complete control over local lattice heating. In this new excitation regime we observed: a) a discontinuity of the QP dynamics which can be attributed to a PIPT from the superconducting to the normal state [30] and b) a new regime of the QP dynamics in the superconducting state, just below the threshold intensity for the PIPT. From the analysis of the latter, we concluded that the number of HFP absorbed during the laser pulse is overhelmingly higher than the QP population, in agreement with the prediction of the $\mathrm{T}_{\text {eff }}$ model [36]. The measured QP dynamics is consistent with both an intermediate $\left(\gamma_{e s c} / \gamma \sim 1\right)$ and a strong bottleneck $\left(\gamma_{e s c} / \gamma \ll 1\right)$ regime of the RTE.

In conclusion, this work constitues a benchmark for future pump-probe experiments on highly anisotropic layered superconductors where the c-axis thermal conduction is strongly suppressed. The main experimental achievement is the possibility to study the optical response of HTSC single crystals in the superconducting state at an arbitrary excitation fluence in the range $\left(10<\phi<600 \mu \mathrm{J} / \mathrm{cm}^{2}\right)$ without significant average heating and with an optimal signal-to-noise ratio. This opens the possibility to investigate experimentally the femtosecond dynamics of the PIPT to the normal state in a wide class of superconductors.

\section{ACKNOWLEDGMENTS}

We thank F. Banfi for the fruitful discussions and for the help in the average heating simulations and sample characterization, S. Pagliara for assistance in the cryogenic setup and A. Damascelli for discussions. This work was supported by the Italian Ministero dell'Istruzione, Università e Ricerca (MIUR). The crystal growth work was done at Stanford University by H. Eisaki, N. Kaneko and M. Greven and was supported by grants from the Department of Energy and the National Science Foundation.

\section{REFERENCES}

1. A. Damascelli, Z. Hussain, and Z. Shen, Rev. Mod. Phys. 75, 473 (2003). 
2. Ø. Fischer, M. Kugler, I. Maggio-Aprile, and C. Berthod, Rev. Mod. Phys. 79, 353 (2007).

3. D. Basov, and T. Timusk, Rev. Mod. Phys. 77, 721 (2005).

4. P. Monthoux, D. Pines, and G. Lonzarich, Nature 450, 1177 (2007).

5. P. Anderson, Nature 317, 1705 (2007).

6. M. Norman, D. Pines, and C. Kallin, Adv. Phys. 54, 715 (2005).

7. S. Han et al., Phys. Rev. Lett. 65, 2708 (1990).

8. S. Brorson et al., Solid State Commun. 74, 1305 (1990).

9. P. Gay et al., J. Low Temp. Phys. 117, 1025 (1999).

10. D. Smith et al., Physica C 341, 2221 (2000).

11. N. Gedik et al., Phys. Rev. B 70, 014504 (2004).

12. N. Gedik, M. Langner, and J.Orenstein, Phys. Rev. Lett. 95, 117005 (2005).

13. N. Gedik, J.Orenstein, R. Liang, D. Bonn, and W. Hardy, Science 300, 1410 (2003).

14. J. Demsar et al., Phys. Rev. Lett. 82, 4918 (1999).

15. D. Mihailovic, and J. Demsar, ACS Symposium Series 730 (1999).

16. J. Demsar et al., Phys. Rev. Lett. 91, 267002 (2006).

17. V. Kabanov, D. Mihailovic, and J. Demsar, Phys. Rev. Lett. 95, 147002 (2005).

18. V. Kabanov, J. Demsar, B. Podobnik, and D. Mihailovic, Phys. Rev. B 59, 1497 (1999).

19. G. L. Carr et al., Phys. Rev. Lett. 85, 3001 (2000).

20. R. A. Kaindl, M. A. Carnahan, and D. S. Chemla, Phys. Rev. B 72, 060510 (2005).

21. Y. H. Liu et al., Phys. Rev. Lett. 101, 137003 (2008).

22. A. Rothwarf, and B. Taylor, Phys. Rev. Lett. 19, 27 (1967).

23. T. Gong et al., Phys. Rev. B 47, 14495 (1993).

24. L. R. Testardi, Phys. Rev. B 4, 2189 (1971).

25. C. Owen, and D. Scalapino, Phys. Rev. Lett. 28, 1559 (1972).

26. W. Parker, Phys. Rev. B 12, 3667 (1975).

27. L. Perfetti et al., Phys. Rev. Lett. 99, 197001 (2007).

28. S. Brorson et al., Phys. Rev. Lett. 64, 2172 (1990).

29. P. B. Allen, Phys. Rev. Lett. 59, 1460 (1987).

30. C. Giannetti et al., submitted to Phys. Rev. B (2008).

31. M. Crommie, and A. Zettl, Phys. Rev. B 43, 408 (1991).

32. K. Krishana, N. P. Ong, Q. Li, G. D. Gu, and N. Koshizuka, Science 277, 83 (1997).

33. M. Schneider et al., Eur. Phys. J. B 36, 327 (2003).

34. A. Junod et al., Physica C 229, 209 (1994).

35. P. Kusar et al., Phys. Rev. Lett. (2008).

36. E. Nicol, and J. Carbotte, Phys. Rev. B 67, 214506 (2003). 